\title{
46,XY Ovotesticular Differences of Sex Development
}

National Cancer Institute

\section{Source}

National Cancer Institute. 46, XY Ovotesticular Differences of Sex Development. NCI

Thesaurus. Code C127173.

The presence of ovarian and testicular tissue in the an individual with 46,XY karyotype.

The anatomical expression of this condition is variable. 